\title{
Vom ,Kalten Terroristen' Zum FriedenSKaiser? ÜBER DIE WENDE IM POLITISCHEN WIRKEN VON OCTAVIAN ZU Augustus
}

\author{
Michael Stahl
}

Technische Universität Darmstadt

Gunther Gottlieb zum 75. Geburtstag

Zusammenfassung: Die politische Laufbahn des ersten römischen Kaisers wirft die Frage auf, wie aus dem seine Gegner erbarmungslos vernichtenden Bürgerkriegskämpfer Octavian nach seinem Sieg der maßstabsetzende Friedensherrscher Augustus werden konnte. Im Kampf um die Macht hatte Octavian jedoch von Beginn an ein weitergehendes Ziel vor Augen, das sich aus seiner Herkunft, seiner Nähe zu Caesar und seiner Verwurzelung in der römischen Tradition erklären lässt und von dem seine Person geprägt war. Res publica restituta ist als Akt historischer Rezeption aufzufassen, der die neue Ordnung mit der der Republik verband und in dem Person und Werk des Monarchen zur Einheit wurden. Dass er dafür einen Weg gehen musste, auf dem er nicht vermeiden konnte, Schuld auf sich zu laden, macht die historische Tragik seines politischen Wirkens aus.

Schlagworte: Octavian/Augustus; Prinzipat; res publica restituta; historische Größe; römische Geschichte

ABStRACT: The political carreer of Rome's first princeps poses the question how the merciless combatant Octavian could become, after his victory in the civil war, Augustus, the bringer of peace. From the beginning, and also during the fight for power, Octavian had an overarching goal in mind, which can be explained by his descent, his close relationship to Caesar and his roots in the Roman tradition, all of which were formative influences on his personality. Therefore, res publica restituta has to be conceptualised as an act of historical reception which 
connected the new order with the republic and in which the person of the monarch and his achievement became one and the same thing. It is the historical tragedy of his political work that he could not avoid becoming guilty in acting out his vision.

Keywords: Octavian/ Augustus; principate; res publica restituta; historical greatness; Roman history

Wir alle haben eine tigerartige Anlage, so wie wir eine himmlische haben sollen (...). Wir alle können nicht wissen, wie wir in den gegebenen Verhältnissen handeln würden, weil wir nicht wissen, welche unbekannten Tiere durch die schreckliche Gewalt der Tatsachen in uns emporgerufen werden können.

Adalbert Stifter

Jeder Aufstieg in der Stufe der Ämter ist nicht ein Schritt in die Freiheit, sondern in die Bindung. Je höher das Amt, desto tiefer die Bindung. Je größer die Amtsgewalt, desto strenger der Dienst. Je stärker die Persönlichkeit, desto verpönter die Willkür.

Hermann Hesse

\begin{abstract}
A m 19. August des Jahres 14 n. Chr. starb Kaiser Augustus in Nola in Kampanien. Er war fast 76 Jahre alt geworden, und sein Tod fiel genau auf jenen Tag, an dem er mehr als ein halbes Jahrhundert zuvor sein erstes Konsulat mit Gewalt errungen hatte, an der Spitze einer irregulären Privatarmee. Was war das für ein Mann, der sich in seinem Sterbezimmer von seiner Ehefrau Livia, mit der er 52 Jahre verheiratet gewesen war, von seinem Stiefsohn Tiberius, den er nie in sein Herz geschlossen hatte, und von den anwesenden Freunden verabschiedete und der sich bei den Umstehenden erkundigt haben soll, ob er das Theaterstück des Lebens bis zum Ende gut gespielt habe? War er wirklich ein Schauspieler, dazu ausersehen, bestimmte Rollen zu spielen, deren Bedeutung allmählich so übermächtig wurde, dass sie ein wahres persönliches Ich daneben nicht mehr zuließen? Oder blieb er zeitlebens der Wolf, der sein grausames Wesen im Laufe der Zeit erfolgreich mit einem feinen Schafspelz zu umhüllen verstand? Oder wie ist mit dem greisen Friedensfürsten, selbst wenn man ihn mit Jochen Bleicken als väterlichen Unsympathen zeichnet, jener „kalte und frühreife Terrorist" zu vereinbaren, als den ihn Ronald Syme einst scharf, aber nicht ohne Grund charakterisierte, jener - noch einmal Syme - „revolutionäre Führer und Abenteurer“, der dann zum „unverhüllten Despoten" wurde? Oder sollen wir tatsächlich glauben, dass jener Octavian,
\end{abstract}


der erbarmungslose Massenmörder von Perusia, der gleiche Mann ist wie jener Augustus, der 38 Jahre später aus Freude und Dankbarkeit gegenüber seinem glückhaften Schicksal im Senat seine Tränen nicht zurückhalten konnte? Das ist das Rätsel, das Augustus unvermeidlich aufgibt, wenn wir sein Leben und Wirken im Ganzen in den Blick nehmen. Mit diesem Rätsel muss sich auseinandersetzen, wer die historische Bedeutung von Augustus beurteilen will.

Ich möchte das Problem ein- und umkreisen, Voraussetzungen, historische und persönliche Bedingungen, Leistungen und Ergebnisse erörtern:

\section{Die Alleinherrschaft als historische Notwendigkeit}

Um zunächst möglichen Mißverständnissen vorzubeugen: Geschichte ist immer ein unabgeschlossener, nicht berechenbarer, d.h. nicht voraussagbarer und also ergebnisoffener Prozess. Die Menschen können nur ihren jeweiligen Möglichkeiten und ihrem Glauben und Dafürhalten gemäß verantwortungsbewusst handeln. Wohin dieses Handeln, zu dem ja stets mehrere Akteure gehören, führen wird, hat niemand sicher in der Hand. Insofern meint die Rede von der historischen Notwendigkeit nicht, dass es so kommen musste, wie es gekommen ist. Dass es eben diesen jungen Octavian gegeben hat, dass er sich militärisch und politisch durchsetzen konnte, dass er einen Weg fand, seine Macht zur dauerhaften Herrschaft werden zu lassen, dass er die lange Zeit bekam, damit sein Werk Wurzeln schlagen konnte, und dass dies schließlich post festum als der Beginn einer neuen Epoche der Weltgeschichte verstanden werden konnte - all dies musste nicht so kommen. Aber dass es so kam, markiert eine einschneidende welthistorische Zäsur, die unser historisches Urteil herausfordert.

Und weil der Prinzipat des Augustus die für uns erkennbar einzige Möglichkeit darstellte, wie die Krise des römischen Gemeinwesens und seines Weltreiches überwunden werden konnte - zum Glück für die Alte Welt wie für die nachmalige Geschichte Europas bis zum Anbruch der Moderne, eben deshalb war die Errichtung der römischen Monarchie eine historische Notwendigkeit. Dass die Christen in ihr nur wenig später einen Ratschluss der göttlichen Vorsehung erblickten und daher das römische Reich in ihre Geschichtstheologie integrierten, ist darüber hinaus höchst bemerkenswert, soll aber hier außer Betracht bleiben.

Die Krise der späten römischen Republik hatte vor allem darin bestanden, dass die stadtstaatlich gebundene republikanische Aristokratie aus sich heraus nicht imstande war, ihren zusammengeraubten, weltumspannenden Machtbereich ordnungsgemäß zu regieren. Die Zustände in den praedia populi Romani waren weitgehend gekennzeichnet durch den Einsatz schierer Gewalt und durch hemmungslose Ausbeutung. Einzelne, ob sie nun Tib. Gracchus, Sulla, Lucullus, Pompeius, Caesar oder Marcus Antonius hießen, 
bedienten sich des Weltreichs als Mittel, um ihre persönliche Machtstellung zu vergrößern, was zwangsläufig nur auf Kosten des inneren Zusammenhalts der Senatsaristokratie gehen musste. Aber keiner dieser Männer hätte anders handeln können. Die zur aristokratischen Existenz notwendig gehörende Wettbewerbsethik forderte von jedem römischen Senator als oberstes Handlungsziel den Erwerb von dignitas, honor und schließlich auctoritas. Zum Erweis von virtus eröffneten sich seit dem Ende des Hannibalkrieges einzelnen Männern bisher ungeahnte Möglichkeiten. Sie wollten jeweils ergriffen sein, sollte ihre Nutzung nicht Konkurrenten und Rivalen überlassen werden. Die damit verbundenen Regelverletzungen und die neuen Handlungshorizonte wurden im 1. Jh.v. Chr. in ihren Folgen so einschneidend, dass sie den politischen Handlungsrahmen irreversibel veränderten. Die Grundlage republikanischer Politik, die ggf. immer wieder neu zu schmiedende Standessolidarität der nobiles, ließ sich nicht wiederherstellen.

Dabei war-vergleichbar der Entstehung griechischer Tyrannenherrschaften - die Anhäufung unerhörter Reichtümer und sozialer Ressourcen und damit von Macht nichts anderes als die Erfüllung eines jeden aristokratischen Traums, sobald diesem die Wirklichkeit einer funktionierenden sozialen Einbindung und Kontrolle nicht mehr Einhalt gebieten konnte. Zuerst entstanden aus den sich neu eröffnenden Gelegenheiten zur Machtakkumulation empfindliche Störungen der politisch-sozialen Balance innerhalb der Mächtigen, schließlich wurden Verlockungen und Herausforderungen, aber auch die aus der Kontrolle der besetzten Räume erwachsenden sachlichen Erfordernisse zu explosiven Sprengsätzen. Ihre Detonation setzte Konflikte und Gewalt frei, unter denen die Nobilität als Führungsschicht des römischen Staates zerrieben und zu einem beträchtlichen Teil physisch ausgelöscht wurde. So hat am Ende das Weltreich seine Eroberer gewissermaßen unter sich begraben.

Das Weltreich war indes kein eigenständiger Akteur. Trotz der katastrophalen Wirkungen, die von ihm auf das innere Gefüge der Herrschermacht ausgingen, stand es zu keiner Zeit als solches zur Disposition, d.h. in der Gefahr, durch Auflehnung und Widerstand verloren zu gehen zu groß waren die militärische Überlegenheit der Römer und ihr unbedingter Wille, jedes Widerstreben und jede potentiell gefährliche Machtballung zu zerschlagen. Doch aus der Perspektive dessen, was sich nachher als möglich erwies, kann man im Sinne der eben definierten historischen Notwendigkeit sagen: Das Untertanengebiet verlangte immer dringlicher nach einer neuen Form der Herrschaft. Sie hatte die Ausbeutung zu beenden, das Agieren Roms in den Provinzen aus den Fehden um Macht und Ehre herauszulösen und den Unterworfenen Frieden, Sicherheit, Wohlstand und eine wie auch immer geartete politische Teilhabe zu bescheren. Sie hatte, anders gesagt, ein Reich erst zu begründen.

Jede Zeit muss die Aufgaben, die ihr gestellt sind, erkennen. Aus jeder Krise findet sich ein Ausweg, auch wenn es lange so scheinen mag, als böte sich vom Bisherigen her keine Alternative. Geht man auf die Grundbedeutung des 
Wortes Krise zurück, so ist der Tod selbstverständlich immer eine Möglichkeit. Aber er stellt in einem normativen Sinn keine Option dar. Das römische Reich hätte zusammen mit der republikanisch-oligarchischen Ordnung Roms zerfallen können, bevor es überhaupt existierte. So war es dem Alexanderreich ergangen, und Sertorius hatte in der nachsullanischen Zeit in Spanien die Möglichkeit einer Sezession und einer Kooperation mit lokalen Eliten für einige Jahre praktiziert. Doch von besonderem historischen Interesse sind Krisenbewältigungen wider die Wahrscheinlichkeit. In ihnen treten plötzlich Menschen auf, die die Zeichen der Zeit zu lesen vermögen, ihre Forderungen erspüren und erkennen und sich in die Pflicht nehmen lassen, etwas Neues zu schaffen.

\section{Octavians Kampf um die Macht - Notwendigkeit und Schuld}

Macht ist für sich genommen nichts Negatives, sondern mit jeder menschlichen Gemeinschaft notwendig gegeben. Wenn wir von Staatlichkeit sprechen, ist die Macht in einer spezifischen Weise konzentriert. Diese staatliche Macht wirkt dann wie ein Magnet, dessen Kräfte die Voraussetzung dafür sind, dass die in jedem Gemeinwesen gegebenen Ordnungsaufgaben in institutionalisierter Form gelöst werden können. Wo Macht das Substrat eines funktionierenden Staates ist, wird sie zur Herrschaft. Ist diese stabil und kann sich über längere Zeit halten, so folgt das daraus, dass die Macht in einer bestimmten Weise ausgeübt wird, nämlich so, dass sie nicht unberechenbar, willkürlich und in rein persönlichem Interesse eingesetzt wird, sondern zum Wohle des Ganzen. Nicht jede Machtstellung führt, wie Herodot dies in seiner Analyse der griechischen Tyrannenherrschaften zu Recht hervorhob, zur moralischen Depravierung ihres Inhabers. Das gilt nur - moderne organisationspsychologische Untersuchungen bestätigen das für nicht gebundene Macht. Die Transformation zur Herrschaft meint eben diese Einhegung von Macht durch das Recht und die ethische (und religiöse) Selbstbindung des Machthabers.

Die Krise der späten römischen Republik bedeutete letztlich die Selbstauflösung des bisherigen Trägers staatlicher Macht, der Senatsaristokratie, und d.h. die unwiederbringliche Zerstörung des lange fraglosen Zentrums der bestehenden Herrschaftsordnung. Jeder Weg aus der Krise konnte daher nur über die Schaffung einer neuen Bündelung von Macht führen, die auch die inzwischen nicht mehr integrierten Akteure, allen voran die Soldaten und die italischen beziehungsweise provinzialen Eliten, auf Dauer einzubeziehen vermochte. Das erste und vordringlichste Ziel jedes zukunftsorientierten politischen Handelns in einer Situation des Mangels an staatlich geordneter Macht musste somit die Erringung und Sicherung einer neuen Machtposition sein. Nach Lage der Dinge, d.h. angesichts des offenkundigen Versagens der kollektiven Machtausübung durch die 
Senatsaristokratie und deren strukturell bedingter Reformunfähigkeit sowie angesichts der Bedürfnisse des weltweiten Machtbereichs und schließlich vor dem Hintergrund zeitgenössisch allein vorstellbarer Alternativen war eine neue Machtkonzentration kaum anders zu verwirklichen, als im Alleinbesitz eines Einzelnen.

Zu dieser Erkenntnis hatte der junge Octavian, während er sich über Jahre hin an der Seite seines nachmaligen Adoptivvaters Caesar aufhielt, aus eigener Anschauung kommen können. Die Umstände, unter denen Caesar am Ende gleichwohl gescheitert ist, haben diese Lehrzeit gerade nicht dementiert, sondern noch einmal eindrucksvoll bestätigt. Denn die Mörder des Diktators waren ohne jede zukunftsweisende politische Perspektive und deshalb am gleichen Tag mit ihrem Latein schon am Ende. Dennoch besetzten für kurze Zeit die Gruppe der Attentäter und dann jene anderen, die gerne in die Fußstapfen Caesars treten wollten, noch die politische Bühne - oder glaubten zumindest, es zu tun. Der Erbe des Ermordeten war im Jahre 44 v. Chr. weit davon entfernt, als der neue Caesar einfach das Werk seines Vaters weiterführen zu können. Keiner konnte also zu diesem Zeitpunkt in ihm mehr sehen als ein politisches Leichtgewicht ohne eigene Position, auch wenn er selbst sich von Beginn an als Teil der neuen, konfliktträchtigen Machtkonstellation betrachtete.

Damit spitzte sich die „Krise ohne Alternative“ neuerlich und rasch zu. Wie hätte vermieden werden können, dass noch einmal Blut zu fließen begann, vielleicht so lange, bis alle Widerstände gegen das Neue weggeschwemmt waren? Wäre es, wie ein Cicero vielleicht geglaubt hatte, wirklich möglich gewesen, das Regime der Nobilität wiederzubeleben, nachdem seit hundert Jahren alle Versuche dazu nur tiefer in die Krise geführt hatten? „Nec vitia nostra, nec remedia pati possumus“, „wir können weder unsere Übel länger ertragen noch die Heilmittel", so diagnostizierte Livius im Rückblick treffend (praef. 9). Und Cicero selbst hatte immer wieder davon gesprochen, dass der Staat zugrunde gegangen sei, „res publica amissa est“. Es war kein Ausweg in Sicht, der aus der bestehenden instabilen Machtlagerung selbst hätte erwachsen können. Alle politischen Vorstellungen, die nach der Ermordung Caesars im Raume standen, die hehren Ideale Ciceros, die patriotischen Appelle der sog. Republikaner um Brutus und Cassius, die triumphalen Skizzen einer alternativen, dem politischen Hellenismus verpflichteten Reichspolitik durch Marcus Antonius, all das hätte die Agonie der alten Ordnung sehr wahrscheinlich nur verlängert. Denn nirgendwo war zu sehen, wie die Grundfrage zu lösen war: wie nämlich die Anarchie zu überwinden und ein stabiles Zentrum staatlicher Macht zu schaffen war.

Kein Weg führte also an dieser Aufgabe vorbei. Ob der junge Octavian sie schon als solche wahrnehmen konnte, wissen wir nicht. Anfangs hat er gewiß einfach in der Überzeugung gehandelt, seinen Adoptivvater und die Ehre der iulischen Familie nicht im Stich lassen zu dürfen und - einer tief in der römischen Moral wurzelnden Pflicht gemäß (Cic. top. 90) - Rache üben zu müssen. Das war in römischen Augen weder abwegig noch moralisch anrüchig. Dass 
Octavian in diesen für ihn zunächst ja vollkommen aussichtslos erscheinenden Konflikt allerdings mit der von Beginn an gezeigten Entschlossenheit, Verve und Zielstrebigkeit gegangen ist, lässt den Verdacht aufkommen, er habe von Anfang an zumindest gespürt, dass sich hinter seinem plausiblen Kampf um die Familienehre noch eine größere Aufgabe verbarg. Zumindest ist ihm wohl sehr schnell klar geworden, dass es darauf ankam, sich selbst erst einmal in eine Machtposition zu bringen, die ihm die Verwirklichung seiner Sohnespflichten überhaupt ermöglichte und ihm eine gewisse Sicherheit verschaffte. Wir können nicht sehen, wann Octavian sich über sein größeres Ziel letztlich klar geworden ist. Doch spricht sein über knapp eineinhalb Jahrzehnte sehr geradliniger und ohne jedes Zögern gegangener Weg dafür, einen hohen Grad von Bewusstheit schon von Beginn an anzunehmen. Wie er dazu kam - darüber können wir nur Vermutungen anstellen. Der Aufenthalt in der Nähe Caesars, sein persönliches Umfeld von Freunden und Beratern, das mag wichtig gewesen sein. Auch weitere persönliche Prägungen spielten eine große Rolle. Darauf wird gleich noch einzugehen sein.

Octavian war sich bei seinem Eintritt in die Politik zweifellos im Klaren darüber, worauf er sich einließ. Und dass dies von ihm verlangte, sich persönlich durchzusetzen, so schnell es ging, ohne jede Rücksicht, auch gegen sich selbst, seine Hände in Blut zu tauchen als - wie Jochen Bleicken es ausgedrückt hat - „ein aus kalter Berechnung mordendes Wesen, ohne eine Spur von Menschlichkeit, als ein ganz gewöhnlicher Terrorist." (S. 149) Gewiss, schon ein flüchtiger Blick auf die Ereignisse legt keinen anderen Schluss nahe, und das Urteil von Ronald Syme ist nicht nur möglich, sondern moralisch aller Ehren wert. Doch ist damit nicht zugleich gesagt, dass Octavian in der Situation, vor die er gestellt war, gar keine andere Wahl hatte, als mit Gewalt zu handeln, wollte er die Voraussetzungen und Mittel dafür schaffen, seinen Vater zu rächen und - wie auch immer dessen politischen Zielen verpflichtet - die alleinige Macht zu erringen, um dem Morden ein Ende zu setzen und Ordnung neu zu schaffen? Mit der Annahme von Caesars Testament tat es der Adoptivsohn seinem Vater nach: wie im Jahre $49 \mathrm{v}$. Chr. wurden fünf Jahre später die Würfel abermals geworfen - nur mit dem Unterschied, dass es bei diesem Mal nicht nur um persönliche dignitas ging. Denn der junge Octavian war, auch wenn er sich rasch eine militärische Basis verschaffen konnte, von seiner persönlichen Statur her nun wirklich keiner der Generäle der alten Ordnung, die ihre persönliche dignitas nicht mehr mit den Ansprüchen und Regularien der Tradition in Deckung bringen konnten. Und er wäre nur dann Aufrührer oder Hochverräter, wenn man die republikanische Staatlichkeit nach der Herrschaft Caesars noch für mehr als eine hehre, aber leblose Idee halten könnte. Doch das ist sehr zu bezweifeln.

Allerdings war Octavian gezwungen, aus der Annahme seiner Adoption die Konsequenzen zu ziehen und sich den Bedingungen zu stellen, die im Jahre 44 v. Chr. galten: mit Caesars Ermordung brach der Bürgerkrieg notwendig wieder aus, und der Griff zu den Waffen war daher auch für den Neuling in 
dieser Arena das Gebot der Stunde. Dass er sich in diesem Ringen behaupten und schließlich durchsetzen würde, konnte allen Beteiligten, nicht zuletzt ihm selbst, nicht anders als hochgradig unwahrscheinlich erscheinen. Allein dieses führt zu der Vermutung, dass es - sollte es nicht bloßer Hazard gewesen, kaum durch begünstigende Faktoren gedeckt - eben noch um etwas anderes ging als um das Aufnehmen eines Machtspiels mit höchst ungewissem Ausgang, das den jungen Mann zu seinem Entschluss bewegte. Dass dieser ihn dereinst zur Alleinherrschaft führen würde, grenzt selbst für den rückschauenden Historiker an ein Wunder. Vielleicht nähme es dieser offenbaren Kontingenz etwas von ihrer Unbedingtheit, wenn man der späteren kaiserlichen Selbstdeutung (RG 1) etwas mehr Realitätsgehalt zubilligen würde.

Nun endet nämlich diese Geschichte des Octavian nicht mit dem Jahre 31 v.Chr. Sie wäre sonst auch nichts als eine weitere Fußnote zu der Geschichte der großen Übeltäter in der Weltgeschichte. „Nemo enim umquam imperium flagitio quaesitum bonis artibus exercuit." "Niemand hat jemals eine Herrschaft, die er durch Verbrechen erlangte, in löblicher Weise ausgeübt." (Tac. hist. 1, 30,1) So urteilt ein Jahrhundert später der Historiker Tacitus mit Blick auf die Wirren des Vier-Kaiser-Jahres 69. Selbstredend: Kein Zweck heiligt die Mittel - vorausgesetzt freilich, man hat eine Wahl. Die Alternative, vor der sich Octavian in den Jahren 44 und 43 v. Chr. sah, war jedoch einzig die zwischen dem Griff zu den Waffen und dem Verzicht auf die Nachfolge Caesars. Besonnenes Abwägen der eigenen Möglichkeiten hätte eher dafür gesprochen, mutlos die Hände in den Schoß zu legen. Wir werden noch hören, dass Tacitus selbst eigentlich die Argumente für eine Sichtweise liefert, die die mehr als mutige Entscheidung des jungen Mannes zudem als die verantwortungsbewusste erscheinen lässt. Und nur weil es genügend überzeugende Beweise dafür tatsächlich gibt, dürfen wir die Bekundungen des Herrschers selbst, Jahrzehnte nach den Ereignissen niedergelegt, nicht als bloße Schönrednerei beiseite tun. Der Retter des Staates sei er gewesen, so Augustus, da er das Feuer der Bürgerkriege ausgetreten habe. Darauf allein kam es gegen Ende seines wundersamen und erfolgreichen Lebens an. Für den Rückschauenden sind freilich die Brandwunden unübersehbar, die für immer an ihm hafteten.

\section{Persönliche Prägungen des Künftigen Princeps}

Unsere Quellen erlauben es nicht, zum Geheimnis der Persönlichkeit des Augustus wirklich vorzudringen. Scheinbar vereinigte dieser Mann zwei Seiten in sich, die gegensätzlicher nicht sein könnten. Dennoch kann man versuchen, dieses Geheimnis so weit wie eben möglich einzukreisen. Dazu sollen nun einige der durch Herkunft und Erziehung gegebenen Prägungen erörtert werden, aus denen heraus Octavian/Augustus gehandelt hat. Diese Prägungen waren - das ist sehr wichtig - selbstverständlich nichts, was nur diesem Manne 
eigen gewesen wäre. Er hat sie vielmehr mit seinen Zeitgenossen geteilt, und sie waren auch für sie verständlich und verbindlich. Was also hat die geistige Persönlichkeit des Augustus geprägt und geformt?

Da ist zum einen seine Herkunft aus einer ritterlichen Familie der italischen Landstadt Velletri, unweit von Rom. Damit befand Octavian sich in einer sozialen und politischen Lage, wie sie für viele seiner Generation typisch war. Aus dem einst mit einer bewundernswerten Herrschaftstechnik an Rom gebundenen Italien war längst, seit $88 \mathrm{v}$. Chr. auch offiziell, das Land römischer Bürger geworden, die zusammen mit den Stadtrömern das Weltreich erobert hatten. Und dennoch waren die Italiker noch nicht so, wie es ihrer inzwischen erreichten Bedeutung entsprach, an der politischen Führung des Staates beteiligt. Die führenden Familien des Landes drängten daher darauf, dass ihre Söhne Senatoren werden konnten und damit Zugang zum inneren Kreis von Macht und Prestige erhielten. Die Geste Caesars, seinen Großneffen, dessen Familie nicht zum stadtrömischen Adel gehörte, doch durch Geldgeschäfte vermögend geworden war, über Jahre zu seinem persönlichen Begleiter zu machen, spricht zumindest für eine vorurteilslose Sichtweise des Diktators. Octavian hat aus ihr die Überzeugung geschöpft, dass Italien künftig so römisch wie die urbs selbst werden und dass seinen Führungsschichten der soziale und politische Aufstieg vollkommen gleichberechtigt ermöglicht werden musste. Der Schwurakt des Jahres 32 v. Chr. - ein Ereignis von großer symbolischer Bedeutung und gar nicht zu überschätzender Tragweite für die Zukunft - hat ihm dafür eine nicht mehr hintergehbare Bestätigung gegeben.

Es war also - und das ist der zweite Gesichtspunkt - in bestimmter Hinsicht ein Blick von außen, mit dem Octavian anders als seine Konkurrenten um die Macht die politischen Bedingungen analysieren konnte, mit denen er es zu tun bekam. Das erlaubte ihm, sich dort vom Zwang der Tradition frei zu halten, wo er sie in ihren konkreten Vorgaben - etwa im "Verfassungsrecht" - den neuen Verhältnissen nicht mehr angemessen empfand. Zugleich jedoch war dieser Blick aus der Distanz dazu geeignet, das Wesentliche zu bestimmen und festzuhalten, was der res publica ihre einstige Stärke verliehen hatte. Und das waren nun einige grundlegende Verhaltensdispositionen, die sich vor allem den Angehörigen der Oberschicht durch ihre Sozialisation einprägten und mit denen auch der junge Octavian vertraut gemacht worden sein dürfte.

Dazu zählte erstens die Orientierung des Handelns an den Normen und Kategorien des Rechts. Die Bedeutung dieser Formierung allen öffentlichen Handelns kommt sogar noch in ihrer pervertiertesten Form zum Ausdruck, der Scheinlegitimation des diktatorischen Terrorregimes der Triumvirn durch ein Volksgesetz. Die Wiederherstellung von Rechtsförmigkeit war dann vor allem auch das wesentliche Motiv für die Art und Weise, wie Octavian im Januar 27 v. Chr. seine Macht in einem ersten Schritt institutionalisiert hat.

Eine andere durch die Tradition beglaubigte Handlungsmaxime verdichtet sich im Begriff der pietas. Es ist damit die Überzeugung der Römer gemeint, dass es zwingende Verpflichtungen gebe, deren bindender Kraft man sich 
nicht entziehen könne und dürfe. Diese Pflichten schufen und regelten die entscheidenden zwischenmenschlichen Verbindungen, die zwischen Vater und Sohn in der familia, also zwischen den Generationen, und die zwischen Patron und Klienten, also zur Gestaltung der gesellschaftlichen Hierarchie. Und schließlich ist pietas die Haltung, mit der der römische Mensch seinen Göttern entgegentrat. Von den ersten Tagen seines öffentlichen Auftretens im Frühjahr 44 v. Chr. an machte Octavian deutlich, wie sehr er sein Handeln aus dem Geist der pietas heraus verstanden wissen wollte: dass er demonstrativ und unmissverständlich zu seinem ermordeten Adotivvater stand und die ihm als Sohn obliegenden Pflichten ernst zu nehmen gedachte, dass er das Testament Caesars vollstrecken ließ, gerade auch in den materiellen Aspekten der patronalen Fürsorge, und dass er sich als Erbe Caesars dessen Klienten gegenüber, und das hieß nach Lage der Dinge in erster Linie Caesars Soldaten und der stadtrömischen plebs gegenüber, in seiner neuen Pflicht als ihr Patron wusste. Seine "Privatarmee" unterschied sich so gesehen in nichts von der Caesars, als dieser den Rubicon überschritten hatte. In einem entscheidenden Punkt gehorchte Octavians Handeln mithin von Beginn an den Geboten der Tradition - auch wenn es hier zunächst noch den Mechanismen der Militärklientel $\mathrm{zu}$ folgen hatte und damit die republikanische Tradition in ihrem noch bis in das zweite Jahrhundert gültigen Inhalt gerade weiter untergrub. Doch auf die Form kam es an, und waren erst die Bedingungen andere geworden, konnte sie mit einem veränderten Inhalt wieder beglaubigt werden. So führt von diesem sich in die Tradition stellenden Handeln Octavians, zu dem natürlich auch die umfangreiche Religionspolitik bereits der Bürgerkriegszeit gehört, wohl doch eine gerade Linie zur pietas als herrscherlicher Kardinaltugend, wie sie auf dem goldenen Schild des Jahres 27 v. Chr. festgehalten ist.

Die Octavian prägende Tradition umfasste noch weitere für das bisherige Selbstverständnis eines römischen Aristokraten zentrale Forderungen: zum einen, dass Aristokrat nur der sein könne, der in Krieg und Politik seine virtus als Mann erweist und dadurch dignitas erwirbt. Ein Grundzug der römischen Aristokraten war ja seit den Anfängen Roms, dass sie sich über ihre Leistung für das Gemeinwesen definierten. Für jeden einzelnen bedeutete das, dass Politik sein wichtigster Lebensinhalt wurde. Es bedarf keines zusätzlichen Beweises, dass Octavian sich diesem Ideal der virtus sein ganzes Leben hindurch voll und ganz verschrieben hatte.

Ein weiterer Aspekt ergibt sich hieraus. Auch die Art und Weise des PolitikMachens hatte sich seit langer Zeit vor allem an einem Grundsatz ausgerichtet: Erfolgreich war der Politiker, dem es gelang, über eine beständige und intensive Kommunikation mit seinesgleichen und der Bürgerschaft Konsens herzustellen und dies auch gebührend herauszustreichen. Was Octavian mit dem consensus universorum von $32 \mathrm{v}$. Chr. gelungen war, ist deshalb nichts kategorial völlig Neues, sondern zumindest auch von diesem traditionellen Ziel eines römischen Politikers her zu verstehen. Und auch diese Linie des 
politischen Handelns von Octavian weist eine evidente Kontinuität auf mit seinem auch als Princeps nicht abreissenden Bemühen um Kommunikation und Konsensstiftung.

Dass Octavian/Augustus schließlich einer anderen traditionell an einen Aristokraten gestellten Forderung unermüdlich nachgekommen ist, nämlich für die römische Machtstellung nach außen hin Sorge zu tragen, auch das liegt offen zutage und muss in seiner Relevanz für das historische Urteil gleich noch eingehender gewürdigt werden.

Die Macht der Tradition formte den Charakter jedes römischen Aristokraten. Diese Kraft war so tief verwurzelt im römischen Leben und Denken, dass sie selbst in den Tagen des Verfalls und der Zerstörung der res publica noch wirksam gewesen ist, ja im ständig beschworenen mos maiorum sogar das einzige war, das noch Geltung beanspruchen durfte. Zweifellos unterwarf sich Augustus dieser Macht, und es wäre vollkommen unglaubhaft, dass er sie erst nach seinem Sieg im Krieg gegen Marcus Antonius plötzlich für sich entdeckt und anerkannt haben sollte. Sie beherrschte seine Persönlichkeit vielmehr von Jugend an, und vielleicht hat er sie - Außenseiter der er war - ernster als seine Altersgenossen aus den berühmten Familien genommen und doch gleichzeitig ein gewisses Maß an Distanz ihr gegenüber einnehmen können. Dieser Abstand gab ihm die Freiheit, sich die Tradition im Rahmen eines veränderten Koordinatensystems noch einmal neu und verändert zu vergegenwärtigen und anzueignen.

In einem Charakterzug überdies verkörperte Octavian/Augustus eine in dieser Tradition liegende und von den Römern selbst für typisch römisch gehaltene Eigenschaft in geradezu vorbildlicher Weise: nämlich in der Hartnäckigkeit und Unbeirrtheit, mit der er trotz mancher Rückschläge sein für richtig erkanntes Ziel verfolgt hat. Dieses Ziel erreichte er nicht durch die Umsetzung einer großen Strategie oder eines fertigen "Masterplans“. Sondern er verfolgte es, indem er stets sich selbst und dem, was in seinen Augen das Römertum ausmachte, treu blieb und in tastenden Schritten immer weiter voranging, indem er vor nichts zurückschreckte, aber auch vorsichtig ausprobierte, Holzwege aufgab und nach besseren Lösungen suchte. Und es brauchte nicht zuletzt diese Fähigkeit, mit langem Atem und festen Grundsätzen sowie der ständigen Bereitschaft dazuzulernen diesen Weg zu gehen, damit am Ende die Krise überwunden und der römische Staat aus dem Geist und der Macht der Tradition heraus neu gegründet werden konnte.

\section{Die scheinbare Wende der Politik Und die Einheit von Person und AufGabe}

Wenn man das konkrete politische Handeln von Octavian/Augustus vor dem Hintergrund der eben geschilderten persönlichen und sozialisationsbedingten Voraussetzungen sowie der allgemeinen politisch- 
gesellschaftlichen Rahmenbedingungen betrachtet, so tritt gegenüber dem eingangs skizzierten Bild zweier scheinbar klar voneinander zu trennender Handlungszusammenhänge, ja Persönlichkeiten - also grob gesagt zwischen Octavian und Augustus - eine viel stärkere Kontinuität der Politik und die innere Einheit der sie tragenden Persönlichkeit hervor.

Es scheint wohl nur so, dass wir seit der Mitte der dreißiger Jahre, also nach dem Jahrzehnt der Erkämpfung der Macht, einen völlig anderen Octavian zu Gesicht bekommen. Gewiss kommt uns auf den ersten Blick seine Politik wie ausgewechselt vor: Sie richtet sich von jetzt an nicht mehr vordringlich auf die zielstrebige und flächendeckende Liquidierung aller Gegner, sondern streckt, zunächst in Italien, die Hand zur Versöhnung aus. Das Ziel ist nun ein primär konstruktives, der wirtschaftliche und soziale Wiederaufbau Italiens und das Ringen um freiwillige Anerkennung und Zustimmung. Aber die Unverzüglichkeit und Sicherheit, mit der Octavian seit dem Sieg über Sextus Pompeius 36v. Chr. diese Wendung vollzogen hat, erweist sich mit Blick auf die tief liegenden Dispositionen und Motive seines Handelns doch nicht länger nur als der raffinierte strategische Schachzug zur Sicherung der Macht. Vielmehr hatte Octavian nunmehr einen Standpunkt erreicht, von dem aus er sich jener Politik widmen konnte, derentwegen er in dieses Spiel auf Leben und Tod überhaupt gegangen oder deren Notwendigkeit ihm im Laufe eines knappen Jahrzehnts immer deutlicher vor Augen getreten war: die Rekonstruktion des römischen Staates und seines Weltreichs - eine Aufgabe, die er nicht zuletzt von seinem genialen, aber allzu selbstbezogenen Adoptivvater geerbt hatte.

Während dieser nur um seiner persönlichen dignitas willen die ganze morsche Welt um sich herum eingerissen hatte, um schließlich als diktatorischer Machthaber, ohnmächtig, weil ohne greifbare Vision für die Zukunft, allein dem gebündelten Hass seiner senatorischen Standesgenossen gegenüberzustehen, sandte Octavian vom erstmöglichen Zeitpunkt an deutliche Signale aus, in welche Pflicht er sich gestellt sah. Und dabei sollte seine Person, anders als bei Caesar nur Mittel, nicht Zweck sein. So verschmolz Octavian/Augustus völlig mit seiner selbstgestellten Aufgabe, er ordnete sich ihr unter und ließ sich an Selbstdisziplin dabei von niemandem übertreffen. Seit dem Jahre 36 v. Chr. tat er in unzähligen symbolischen Akten und Bildern immer wieder kund, dass er vor allen anderen die Verantwortung für diese Aufgabe trage und dass er sich dessen stets bewusst sei. Daher versteckte er sein asketisches Arbeitsethos auch keineswegs - auch dies ein wichtiger Teil des traditionellen römischen Selbstbildes.

\section{Augustus und Seine Leistung vor der Geschichte}

Angenommen, Octavian und Antonius wären zwischen 44 und $42 \mathrm{v}$. Chr. gefallen oder ermordet worden. Auch dann wäre der republikanischen Ordnung sehr wahrscheinlich kein langes Leben mehr beschieden gewesen. Es 
gibt keinen Grund anzunehmen, dass nicht über kurz oder lang neue Figuren in das Spiel um die Macht im Staat, und das heißt um die Ressourcen eines Weltreichs, eingetreten wären. Denn die schiere Existenz der römischen Weltstellung in ihrer bisherigen Form lud dazu förmlich ein. Ja vielleicht wäre sogar diese im Laufe der Zeit in Gefahr geraten und auseinandergebrochen - sei es, dass ihr durch eine Fortsetzung der Ausplünderung allmählich die materielle Grundlage weggebrochen wäre, sei es durch ein Arrangement der Mächtigen, wie es zwischen 43 und $33 \mathrm{v}$. Chr. bestand - und im übrigen einen langen Schatten bis ins 3. nachchristliche Jahrhundert und die Zeit danach vorauswirft. In jedem Falle steuerte die seit fast hundert Jahren endemische Krise auf eine grundlegende Änderung der römischen Republik zu, auch wenn sich ihre Protagonisten möglicherweise noch ein paar Jahre oder Jahrzehnte im bisherigen Rahmen und mit anhaltenden Konflikten weiter hätten behaupten können. Die fundamentale Krise des römischen Gemeinwesens zu lösen und den Fortbestand seiner Machtstellung nach außen zu sichern, das war indessen nur möglich durch eine Beendigung und dauerhafte Klärung des inneren Machtkampfes und d.h. die Etablierung einer wie immer gearteten Alleinherrschaft.

Beides ist Octavian/Augustus gelungen, und beides haben schon die Zeitgenossen so gesehen. Dafür spricht nicht zuletzt die schon unter Augustus klare und sich im weiteren rasch verfestigende Akzeptanz des Prinzipats. Im Hinblick auf die Unausweichlichkeit der Alleinherrschaft referiert Tacitus das einschlägige, freilich von einer Minderheit im Senat noch nicht geteilte Urteil: Es habe kein „anderes Heilmittel für die Zerrissenheit des Vaterlandes gegeben, als dass es von einem Mann regiert wurde" (ann. 1,9,4). Die Gestaltung der Monarchie als Prinzipat beruht dabei nicht nur auf der Schaffung eines komplexen, aber stabilen rechtlichen Rahmens. Für sich genommen könnte dieser nämlich durchaus lediglich als Bemäntelung einer de facto weiter bestehenden Tyrannis angesehen werden. Erst die patronale Selbstbindung des Princeps, sein persönliches Ethos, formuliert und kommuniziert in einem reich differenzierten und hoch entwickelten kulturellen System von Bildern, Texten, Praktiken und Ritualen, erzeugte jene auctoritas, durch die diese persönliche Herrschaft allein Bestand hatte. Von diesem Wert ist der augusteische Klassizismus in allen seinen Facetten durchdrungen.

So ist etwa, um nur eine einzelne Facette herauszugreifen, die bewusste Stilisierung besonders in der Formgebung des Herrscherbildes, nicht - wie etwa im Falle von Mussolini oder Hitler - die Aufrichtung einer beliebigen Schaufassade zur Camouflage der hässlichen Kehrseite des Regimes. Sie ist vielmehr das Ergebnis einer ästhetischen Entscheidung, die darauf abzielt, im Schönen die Überwindung des Abgründigen zum Vorschein zu bringen. So signalisiert die Schönheit der Statue von Primaporta, die sich das ideale Menschenbild der griechischen Hochklassik aneignet, jene - in den Worten Foucaults - „Sorge um sich selbst", die schon seit dem Hellenismus die Voraussetzung für die politische Handlungsfähigkeit der Oberschichten bildete. 
Stoßen wir damit womöglich sogar auf einen Zug der realen Persönlichkeit des Augustus? Zumindest erkennen wir in seiner Bildnisrepräsentation ein Ideal, das ihm wichtig war. Gehört es zu jenen Lebensrollen, von denen er auf seinem Totenbett gesprochen haben soll? Und müssen wir diese Rollen nicht ernstnehmen als stimmigen und kohärenten Zusammenhang von Lebensformen und Handlungszielen, aus denen sich sein herrscherliches Selbstverständnis bildete? Suetons Worte vom beifallheischenden Schauspieler in der Komödie des Lebens werden jedenfalls meist allzu vordergründig als Aufforderung verstanden, unter die Theatermasken zu schauen, um deren Träger in seinem ungeschminkten Charakter zu entdecken und ihn im wörtlichen Sinne zu entlarven.

Nehmen wir die grandiose Inszenierung aber ernst, dann wird auch die innere Geschlossenheit des politischen Wirkens dieses Mannes evident: Sowohl auctoritas, der Kardinalbegriff der Monarchie, wie die Rechtsformen, in denen sich das monarchische Handeln zu vollziehen hatte, weisen zurück auf Grundpfeiler der gesellschaftlichen und staatlichen Ordnung der Republik. Res publica restituta - wie Augustus diese Tradition in einem veränderten Rahmen neu vergegenwärtigt und seinem Prinzipat anverwandelt hat, war nichts weniger als eine Meisterleistung historischer Rezeption. Das oft so bezeichnete „Bündnis mit der Tradition“, das Octavian schloss, um Augustus zu werden, meint diesen genuinen Akt „schöpferischer Restauration“ (Rudolf Borchardt). Mit ihm entstand in der augusteischen Epoche ein Geschichtsbild, das seiner Gegenwart aus der Tradition die entscheidenden Werte zur Orientierung auf die Zukunft beschrieb.

Gewiss wurde von den Handelnden nicht wahrgenommen oder reflektiert, wie hier aus dem Alten etwas Neues geschöpft wurde, sondern man verstand es als Vergewisserung des mos maiorum und als Rückkehr zu ihm. Als solches spiegelt es auf der persönlichen Ebene aber auch die Einheit, die den greisen Friedensfürsten mit dem jungen Mann verbindet, den er an den Beginn seiner Lebensbilanz stellt und zu dessen frühesten intellektuellen Erfahrungen die Einsicht gehört haben dürfte, wie hohl die exempla maiorum angesichts der Realität klangen, dem ihre Botschaften aber deswegen nicht gleichgültig wurden. Indem er sie für den Bau der neuen Ordnung fruchtbar machte, erweisen sich auch von daher Person und Werk als ein zusammenhängendes Ganzes.

Dieser Prinzipat war nun die Grundlage und Vorraussetzung dafür, dass Augustus mit Blick nach außen völlig neue Wege beschreiten konnte. Ja, man kann mit gutem Recht sagen: Das Imperium Romanum existiert recht eigentlich erst seit und durch Augustus.

Verleidet waren ihnen (sc. den Provinzen) Senats- und Volksherrschaft wegen der Machtkämpfe der führenden Männer und der Habsucht der Beamten; schwach war der Schutz der Gesetze, die durch Eigenmächtigkeit, politische Umtriebe, vor allem durch Bestechung unwirksam gemacht wurden.

(Tac. ann. 1, 2,2) 
So beschreibt jener Tacitus, dem die Errichtung der Monarchie zutiefst zuwider war - ein deswegen in dieser Sache unverdächtiger Zeuge -, die Zustände im Weltreich der römischen Republik und beschönigt dabei nichts. Wie Italien so waren daher, noch einmal Tacitus an der gleichen Stelle, „auch die Provinzen (...) jener Ordnung der Dinge nicht abgeneigt." Gemeint ist ihre Unterstellung unter die Obhut des Princeps. Daraus sprudelte für den Monarchen denn auch jener unerschöpfliche Quell, aus dem er Rechtfertigung für seine Herrschaft und Segen wie Zustimmung seiner schutzbefohlenen Untertanen schöpfen konnte. Denn, so referiert Tacitus das Urteil der Nachwelt, als Augustus der Herr der Welt geworden war,

habe er den Staat neu begründet; durch den Ozean oder durch weit entlegene Ströme sei das Reich geschützt; Legionen, Provinzen, Flotten, alles sei untereinander straff verbunden; Recht gelte gegenüber den Bürgern, Rücksicht gegenüber den Bundesgenossen; (...).

(Tac. ann. 1, 9,5)

Die Befriedung der Welt begann noch vor Actium in Italien, und als der militärische Sieg errungen war, wurde der Friede des Augustus, die pax Augusta, die Parole für die Schaffung der neuen Ordnung im ganzen Reich. Sie umfasste auch die Restitution und Gewährleistung der gesellschaftlichen Verhältnisse in den Provinzen und führte zu Rechtssicherheit und Gerechtigkeit. Diese neuen Rahmenbedingungen für das Leben im Reich wurden binnen weniger Jahrzehnte, ja zuweilen sogar weniger Jahre überall spürbar wirksam. Sie brachten einen bis dato nicht gekannten wirtschaftlichen Aufschwung, der breiten städtischen Bevölkerungsschichten einen beispiellosen Wohlstand bescherte. Und mit ihm schließlich eine einzigartige kulturelle Blüte, die nicht bloß auf die Hauptstadt der Welt beschränkt blieb. Einige der schönsten Kunstwerke, die die Antike dem Erbe der Menschheit geschenkt hat, entstammen der augusteischen Epoche. Wie hätten sie entstehen können in einem Klima der Verstellung und Heuchelei? Wer die Prinzipatsverfassung nur für eine Theaterfassade zur Verschleierung von Tyrannenmacht hält, degradiert die augusteische Kunst zur hohlen Kulisse. Es sind also nicht zuletzt deren Botschaften des Schönen, die den Charakter der neuen Ordnung beglaubigen - nicht um den dunklen Grund von Krieg, Gewalt und moralischer Schuld einfach vergessen $\mathrm{zu}$ machen, sondern indem sie für deren Überwindung zeugen. So wurde die pax Romana im Zeichen des Princeps zum Signum einer neuen Epoche, die als erste jene Generation zwischen etwa 20 v. Chr. und $14 \mathrm{n}$. Chr. erleben durfte und deren Erfahrungen sich bei aller freiwilligen Rückbindung an die große Tradition Roms doch fundamental abhoben von der gesamten Geschichte der zurückliegenden Jahrhunderte.

Die Zeugnisse aus Italien und dem Reich dafür, dass auch den Zeitgenossen bewusst war, was sie da erlebten, sind zahlreich und dokumentieren die unverstellte Dankbarkeit gegenüber dem Mann, der der Urheber dieser 
Zustände war. Sie konnten allen gar nicht anders erscheinen, denn als Wiederkehr des goldenen Zeitalters. Die Dichter und Künstler haben dieses Empfinden in gültigen Bildern zum Ausdruck gebracht. Und dass man von ihnen jeden Vorwurf fernhalten sollte, es hätte ihnen dabei irgendein „Propagandaminister" die Feder oder den Meißel geführt, dafür gibt es Belege bis in das banalste Alltagsleben der breiten Bevölkerung hinein. Ich erinnere lediglich an ein Beispiel aus der Zeit kurz vor dem Tode des Kaisers, das der Biograph Sueton überliefert:

Als er (nämlich Augustus) gerade an der Bucht von Puteoli vorbeifuhr, hatten ihn Passagiere und Seeleute eines eben angekommenen Schiffes aus Alexandria, weiß gekleidet, bekränzt und Weihrauch verbrennend, mit Wünschen für ein glückliches Ergehen und mit grenzenlosen Lobpreisungen überhäuft: Durch ihn würden sie leben, durch ihn zur See fahren, durch ihn auch Freiheit und Wohlstand genießen.

(Suet. Aug. 98,2)

Kein Zweifel: Das war trotz aller Schuld, die dieser Mensch auf sich zu laden hatte, und trotz allen persönlichen Unglücks, das auch ihm widerfuhr, ein gelungenes Leben. Augustus war es vergönnt, sein Werk erfolgreich zu vollenden, so erfolgreich, dass es das Gehäuse bildete, in dem die römische Geschichte mindestens für weitere gut zweieinhalb Jahrhunderte ihren Platz finden konnte. Erfolg, Gelingen, Glück in der Geschichte - was kann das heißen? Es kann doch nur eine Ordnung beschreiben, die es möglichst vielen Menschen möglichst lange erlaubt, unter friedlichen und prosperierenden Verhältnissen zu leben bei möglichst geringer Verletzung elementarer ethischer Normen (auch wenn diese in der konkreten Formulierung immer zeitabhängig bleiben) und mit möglichst breiter Identifikation mit ihrer Gesellschaft und Gemeinschaft. Gemessen daran war Augustus einer der erfolgreichsten Herrscher der Geschichte, und es ist wenig erstaunlich, dass alle späteren bis in die Neuzeit immer wieder auf ihn zurückgekommen sind.

Das scheinbar Paradoxe an der Geschichte des Augustus und der Begründung des Prinzipats, auf das ich eingangs aufmerksam gemacht hatte, lässt sich gewiss nicht völlig auflösen. Aber es fordert ein Urteil. Dieses kann wohl kaum darin liegen, mit abwiegelnden Kompromissformeln zu hantieren wie etwa: „die Leistung rühmen, die Person hassen“; oder "hinsichtlich des Charakters umstritten, als Herrschergestalt groß". Wo alles so auf die persönliche Leistung eben dieses Einzelnen ankam wie bei der Konstituierung der Princepsstellung, ist eine solche Trennung nicht statthaft. Und auch die Spaltung der Persönlichkeit zwischen dem Terroristen und dem Friedenskaiser ist mehr als problematisch. Was den vermeintlichen Terroristen angeht, so sollte man eine klare Unterscheidung beachten: Einerseits gibt es einen ideologisch begründeten oder verbrämten, in Wahrheit allein einer Wahnvorstellung oder der persönlichen Macht einer Gruppe oder eines einzelnen dienenden Terror 
à la Auschwitz oder Archipel GULAG. Etwas qualitativ anderes scheint mir dagegen die Anwendung gewiss äußerster Gewaltmittel zu sein, zu denen Octavian bei seinem Aufstieg zu seiner Machtstellung zweifellos gegriffen hat. Hier ist bestimmt nichts zu beschönigen oder zu entschuldigen.

Aber nur wer aus den Bedingungen der Zeit heraus plausibel zu zeigen vermöchte, dass es einen anderen Weg als den Krieg mit all seinen schmutzigen Begleiterscheinungen gegeben hätte, um Macht als Grundlage und Voraussetzung für eine neue Ordnung wiederherzustellen, nur der hätte ein Recht, Octavians ohne Zweifel konsequenter und erbarmungsloser Gewaltanwendung die Legitimität abzusprechen. Sie liegt in der nach Erreichung des Zieles sofort eingeleiteten Politik der Friedensstiftung und Versöhnung. Sollten sich also, nach einer Formulierung von Marion Giebel, "die Extreme berühren und eines ohne das andere nicht möglich sein?" Oder, wie Friedrich Vittinghoff 1959 schrieb: „Der Caesar der Ächtungen trug in sich die Möglichkeit, auch der ,Augustus', der ,Vater des Vaterlandes' zu werden." Augustus' „zweite Politik“ jedenfalls war nicht bestimmt von einer Ideologie der Züchtung eines neuen und besseren Menschen, sondern von der Vision besserer Zustände, die sich einstellen, sobald die Menschen sich ihrer moralischen Kräfte besinnen können, wie Herodot einst für die Athener konstatierte $(5,78)$. Eben dies ist gemeint, wenn man der Politik des Augustus zu Recht eine humanistische Ausrichtung zuspricht.

Der Sozialphilosoph Alexander Rüstow hat 1941 in einer Besprechung von Symes „Römischer Revolution“ bemerkt, dass der „einzige Zweck, der die Mittel der Gewaltanwendung wirklich zu heiligen vermöge“, „der universale Dauerfriede" oder wenigstens die Annäherung an ihn sei. Dieses Ziel habe Augustus für die von ihm beherrschte Welt - und das war damals die "Welt" - nahezu erreicht. Und so pries der Engel des Herrn, den der Evangelist Lukas vom Himmel herabsteigen ließ, den Frieden auf Erden ebenso wie jener Engel der Geschichte, den vor 120 Jahren Theodor Mommsen am Anfang des 5. Bandes seiner „Römischen Geschichte“ beschwor:

(...) das römische Kaiserregiment (...) hat in seinem Kreise, den die, welche ihm angehörten, nicht mit Unrecht als die Welt empfanden, den Frieden und das Gedeihen der vielen vereinigten Nationen länger und vollständiger gehegt, als es irgendeiner anderen Vormacht je gelungen ist. [Das vorzügliche Buch Herfried Münklers über die Imperien im Vergleich kommt heute zu keinem anderen Ergebnis! Zusatz des Verf.] In den Ackerstädten Afrikas, in den Winzerheimstätten an der Mosel, in den blühenden Ortschaften der lykischen Gebirge und des syrischen Wüstenrandes ist die Arbeit der Kaiserzeit zu suchen und auch zu finden. Noch heute gibt es manche Landschaft des Orients wie des Okzidents, für welche die Kaiserzeit den an sich sehr bescheidenen, aber doch vorher wie nachher nie erreichten Höhepunkt des guten Regiments bezeichnet [Auch dieses Urteil besitzt nach wie vor volle Gültigkeit, und die Europäer sollten es sich zu ihrer Schande eingestehen! Zusatz des Verf.]; und wenn einmal ein Engel des Herrn die Bilanz aufmachen sollte, ob das von Severus Antoninus 
beherrschte Gebiet damals oder heute mit größerer Humanität regiert worden ist, ob Gesittung und Völkerglück im allgemeinen seitdem vorwärts- oder zurückgegangen sind, so ist es sehr zweifelhaft, ob der Spruch zugunsten der Gegenwart ausfallen würde.

(Theodor Mommsen: Römische Geschichte Bd. 5, Einleitung)

Das scheinbare Paradox des Augustus, das Rätsel seiner Persönlichkeit wird eine Herausforderung für die historische Urteilsbildung bleiben. Wir begegnen hier in extremer Form Bedingungen politischen Handelns, wie sie vergleichbar in der Geschichte immer wieder auftreten können. Nicht jedem Staatsmann ist es vergönnt, unter so glücklichen Umständen wirken zu können wie einem Solon von Athen, der der Gewalt entsagen und allein auf die Kraft der überzeugenden Rede vertrauen durfte. Viel häufiger kommt es vor, dass politisches Handeln der Notwendigkeit nicht entgehen kann, durch Gewalt und Krieg Schuld auf sich zu laden. Es gibt Situationen, in denen ein Staatsmann keine Wahl hat, als das nach seiner Abwägung im Hinblick auf ein höheres Ziel geringere Übel in Kauf zu nehmen. Früher hat man das zu Recht die tragische Dimension der Geschichte genannt. Augustus jedenfalls wird man trotz oder gerade wegen aller Tragik seines Lebens das Epitheton der geschichtlichen Größe nicht verwehren können, auch wenn er, weil sich einmal vergossenes Blut nicht aus der Erinnerung tilgen lässt, nie ein "Großer" genannt wurde. Denn seine Wirklichkeit gewordene Vision von Monarchie und Reich hält trotz mancher, insbesondere jüngerer Missdeutungen - auch heute, wo wir vielleicht auf der Schwelle zu einem postdemokratischen und postmodernen Zeitalter stehen, Botschaften für die geschichtliche Orientierung bereit.

\section{LITERATUR}

Bleicken, Jochen: Augustus. Eine Biographie. Berlin 1998. TB Reinbek 2010.

Bringmann, Klaus: Augustus. Darmstadt 2007.

Castritius, Helmut: Der römische Prinzipat als Republik. Husum 1982.

Dahlheim, Werner: Geschichte der römischen Kaiserzeit. München ${ }^{2} 2003$.

Ders.: Julius Caesar. Die Ehre des Kriegers und die Not des Staates. Paderborn u.a. 2005.

Ders.:Augustus. Aufrührer, Herrscher, Heiland. München 2010.

DeININGER, JÜRGEN: Zur Kontroverse über die Lebensfähigkeit der Republik in Rom. - In: P. Kneißl / V. Losemann (Hrgs.): Imperium Romanum. FS Karl Christ z. 75. Geburtstag. Stuttgart 1998, S. $123 \mathrm{ff}$. 
Flaig, EgON: Den Kaiser herausfordern. - In: HZ 253, 1991, S. $371 \mathrm{ff}$.

Galinsky, Karl: Augustan Culture. An Interpretative Introduction. Princeton 1996.

Giebel, Marion: Augustus. Reinbek 1984.

Heinze, Richard: Auctoritas (1925). - In: Ders.: Vom Geist des Römertums. Ausgewählte Aufsätze. Darmstadt 1972, S. 43ff.

Ders.: Die augusteische Kultur. Leipzig ${ }^{3} 1939$.

Lotze, Detlef: Märchenprinz und Tyrann: der Erbe Caesars. - In: Klio 67, 1985, S. 6ff.

Mause, Michael: Augustus: „Friedensfürst“ in einer unruhigen Zeit. In: Klio 81, 1999, S. $142 \mathrm{ff.}$

SCHMitTHenNer, WAlter: Caesar Augustus - Erfolg in der Geschichte. - In: Saeculum 36, 1985, S. $286 \mathrm{ff}$.

Simon, Erika: Augustus. Kunst und Leben in Rom um die Zeitenwende. München 1986.

Stahl, Michael: Tyrannis und das Problem der Macht. Die Geschichten Herodots über Kypselos und Periander von Korinth, - In: Hermes 111 (1983), S. 202-220.

Ders.: Botschaften des Schönen. Kulturgeschichte der Antike. Stuttgart 2008.

Ders.: Auctoritas und Charisma: Die Bedeutung des Persönlichen in der Herrschaft des Augustus. - In: Potestas 1, 2008, S. 23ff.

Strothmann, Meret: Augustus - Vater der res publica. Zur Funktion der drei Begriffe restitutio - saeculum - pater patriae im augusteischen Prinzipat. Stuttgart 2000.

Syme, Ronald: Die Römische Revolution. Neuausgabe Stuttgart 2003 (1939).

Wallace-Hadrill, Andrew: Rome's Cultural Revolution. Cambridge 2008.

Walter, Uwe: Struktur, Zufall, Kontingenz? Überlegungen zum Ende der römischen Republik. - In: K.-J. Hölkeskamp (Hrsg.): Eine politische Kultur (in) der Krise? München 2009, S. $27 \mathrm{ff}$.

Welwei, Karl-Wilhelm: Caesars Diktatur, der Prinzipat des Augustus und die Fiktion der historischen Notwendigkeit. - In: Gymnasium 103, 1996, S. 477ff.

Yavetz, Zvi: The personality of Augustus. - In: K. A. Raaflaub / M. Toher (Eds.): Between Republic and Empire. Berkely u.a. 1990, S. $21 \mathrm{ff}$. 